\title{
Extending Polynomials in Maximal and Minimal Ideals
}

\author{
by
}

\author{
Daniel CARANDo and Daniel GALICER
}

\begin{abstract}
Given a homogeneous polynomial on a Banach space $E$ belonging to some maximal or minimal polynomial ideal, we consider its iterated extension to an ultrapower of $E$ and prove that this extension remains in the ideal and has the same ideal norm. As a consequence, we show that the Aron-Berner extension is a well defined isometry for any maximal or minimal ideal of homogeneous polynomials. This allows us to obtain symmetric versions of some basic results of the metric theory of tensor products.
\end{abstract}

2010 Mathematics Subject Classification: 46G25, 46A32, 46B28, $47 \mathrm{H} 60$.

Keywords: extension of polynomials, polynomial ideals, symmetric tensor products of Banach spaces.

\section{$\S 1$. Introduction}

Aron and Berner showed in [1] how to extend continuous polynomials (and some holomorphic functions) defined on a Banach space $E$ to the bidual $E^{\prime \prime}$. Some time later, Davie and Gamelin 8 proved that this extension preserves the norm. This fact is crucial to showing that some holomorphic functions defined on the unit ball of $E$ can be extended to the ball of $E^{\prime \prime}$. A natural question is whether a polynomial ideal is closed under the Aron-Berner extension and, also, if the ideal norm is preserved by this extension. This is easy for nuclear and approximable polynomials, and it is also known to hold, for example, for integral polynomials [7, for extendible polynomials [4, and for the ideal of polynomials that are weakly continuous on bounded sets 21 among others. However, some polynomial ideals

Communicated by H. Okamoto. Received October 28, 2009. Revised February 2, 2010.

D. Carando: Departamento de Matemática - Pab I, Facultad de Cs. Exactas y Naturales, Universidad de Buenos Aires, (1428) Buenos Aires, Argentina, and CONICET;

e-mail: dcarando@dm.uba.ar

D. Galicer: Departamento de Matemática - Pab I, Facultad de Cs. Exactas y Naturales, Universidad de Buenos Aires, (1428) Buenos Aires, Argentina, and CONICET;

e-mail: dgalicer@dm.uba.ar

(C) 2010 Research Institute for Mathematical Sciences, Kyoto University. All rights reserved. 
are not closed under Aron-Berner extension (for example, the ideal of weakly sequentially continuous polynomials).

Floret and Hunfeld showed that there is an extension, the so called uniterated Aron-Berner extension, which is an isometry for maximal polynomial ideals [17]. Although it is easy to prove properties of this uniterated extension, it is hard to compute, since its definition depends on an ultrafilter. On the other hand, the (iterated) Aron-Berner extension is not only easier to compute, but also has a simple characterization that allows one to check if a given extension of a polynomial is actually its Aron-Berner extension [24. Moreover, this extension is more widely used in the study of polynomials and analytic functions (for example, it allows a description of the spectrum of the algebra of bounded type analytic functions [2]).

Extensions of polynomials to ultrapowers were first studied by Lindström and Ryan in [20] and by Dineen and Timoney in [12], where they show that different extensions preserve the uniform norm. In [17] the authors further developed some of these ideas and proved that maximality and ultrastability are equivalent for a polynomial ideal [17, Theorem 3.2]. Therefore, for maximal ideals, the uniterated extension to the ultrapower turns out to be an isometry.

One of the aims of this note is to prove that the Aron-Berner extension is a well defined isometry on maximal and on minimal ideals. More generally, we study the extension of a polynomial on a Banach space $E$ to some ultrapower $(E)_{\mathfrak{U}}$, and show the following: if $\mathcal{Q}$ is a maximal or a minimal ideal of $n$-homogeneous polynomials and $P \in \mathcal{Q}(E)$, then the iterated extension $\bar{P}$ of $P$ to the ultrapower $(E)_{\mathfrak{U}}$ is in $\mathcal{Q}\left((E)_{\mathfrak{U}}\right)$, and $\|P\|_{\mathcal{Q}(E)}=\|\bar{P}\|_{\mathcal{Q}\left((E)_{\mathfrak{U}}\right)}$. As a contribution to the metric theory of symmetric tensor products, we derive the symmetric versions of the Extension Lemma and the Embedding Lemma [9, 13.2 and 13.3] (which in the non-symmetric setting are much easier to prove). These are Corollaries 3.4 and 3.5 below.

The article is organized as follows. In Section 2 we describe some basic properties of ultrapowers and define the extensions mentioned above. In Section 3 we prove, following $[8]$ and using the representation theorem for maximal polynomial ideals, the results stated in the previous paragraph.

We refer to 13, 14, 15, 16, 17, for the theory of symmetric tensor products and polynomial ideals.

\section{$\S 2$. Preliminaries}

Throughout the paper, $E$ and $F$ will be Banach spaces, $E^{\prime}$ the dual space of $E$, $\kappa_{E}: E \rightarrow E^{\prime \prime}$ the canonical embedding and $B_{E}$ the open unit ball of $E$. We will denote by $\operatorname{FIN}(E)$ the class of all finite-dimensional subspaces of the Banach space $E$. 
We now recall some basic properties of ultrapowers. The reader is referred to [18, 19] for further details. Let $\mathfrak{U}$ be an ultrafilter on a set $I$. Whenever the limit with respect to $\mathfrak{U}$ of a family $\left\{a_{\mathfrak{i}}: \mathfrak{i} \in I\right\}$ exists, we denote it by $\lim _{\mathfrak{i}, \mathfrak{U}} a_{\mathfrak{i}}$. For a Banach space $E,(E)_{\mathfrak{U}}$, the ultrapower of $E$ with respect to the ultrafilter $\mathfrak{U}$, consists of the classes of elements of the form $z=\left(z_{\mathfrak{i}}\right)_{\mathfrak{U}}$, with $z_{\mathfrak{i}} \in E$ for each $\mathfrak{i} \in I$, where the norm of $\left(z_{\mathfrak{i}}\right)$ is uniformly bounded, and where we identify $\left(z_{\mathfrak{i}}\right)$ with $\left(y_{\mathfrak{i}}\right)$ if $\lim _{i, \mathfrak{U}}\left\|z_{\mathfrak{i}}-y_{\mathfrak{i}}\right\|=0$. The space $(E)_{\mathfrak{U}}$ is a Banach space under the norm

$$
\left\|\left(z_{\mathfrak{i}}\right)_{\mathfrak{U}}\right\|=\lim _{\mathfrak{i}, \mathfrak{U}}\left\|z_{\mathfrak{i}}\right\| .
$$

We may consider $E$ as a subspace of the ultrapower $(E)_{\mathfrak{U}}$ by means of the canonical embedding $h_{E}: E \hookrightarrow(E)_{\mathfrak{U}}$ given by $h_{E} x=\left(x_{\mathfrak{i}}\right)_{\mathfrak{U}}$ where $x_{\mathfrak{i}}=x$ for all $\mathfrak{i}$.

Let us now define the ultrapower of an operator. If $T: E \rightarrow F$ is a bounded linear operator, the ultrapower operator with respect to the ultrafilter $\mathfrak{U}$ will be the operator from $(E)_{\mathfrak{U}}$ to $(F)_{\mathfrak{U}}$ defined according to the rule $\left(z_{\mathfrak{i}}\right)_{\mathfrak{U}} \mapsto\left(T z_{\mathfrak{i}}\right)_{\mathfrak{U}}$. We will denote this operator $(T)_{\mathfrak{U}}$. It can be seen that $\left\|(T)_{\mathfrak{U}}\right\|$ is $\|T\|$.

We will need a special property of ultrapowers [18, Proposition 6.1], 19, Satz 4.1]:

Proposition 2.1 (Local determination of ultrapowers). Let $E$ be a Banach space and $M \in \operatorname{FIN}\left((E)_{\mathfrak{U}}\right)$. For each $\mathfrak{i} \in I$ there exists an operator $R_{\mathfrak{i}} \in \mathcal{L}(M, E)$ such that

(1) $z=\left(R_{\mathfrak{i}} z\right)_{\mathfrak{U}}$ for all $z \in M$.

(2) $\left\|R_{\mathfrak{i}}\right\| \leq 1$ for all $\mathfrak{i} \in I$ and there is an $\mathcal{U} \in \mathfrak{U}$ with $\left\|R_{\mathfrak{i}}\right\|=1$ for all $\mathfrak{i} \in \mathcal{U}$.

(3) For all $\varepsilon>0$ there is an $\mathcal{U}_{\varepsilon} \in \mathfrak{U}$ such that the inverse $R_{\mathfrak{i}}^{-1}: R_{\mathfrak{i}}(M) \rightarrow M$ exists and $\left\|R_{\mathfrak{i}}^{-1}\right\| \leq 1+\varepsilon$ for all $\mathfrak{i} \in \mathcal{U}_{\varepsilon}$.

We shall only use (1) and the first part of (2).

There are different ways of extending polynomials from a Banach space to an ultrapower. Two approaches are the iterated and the uniterated extensions. Let $(E)_{\mathfrak{U}}$ be an ultrapower of a Banach space $E$. For a continuous $n$-linear function $\Phi$ on $E$ we define an $n$-linear map $\bar{\Phi}$ on $(E)_{\mathfrak{U}}$ by

$$
\bar{\Phi}\left(z_{1}, \ldots, z_{n}\right)=\lim _{\mathfrak{i}_{1}, \mathfrak{U}} \ldots \lim _{\mathfrak{i}_{n}, \mathfrak{U}} \Phi\left(z_{\mathfrak{i}_{1}}^{(1)}, \ldots, z_{\mathfrak{i}_{n}}^{(n)}\right)
$$

for $z_{j}=\left(z_{\mathfrak{i}_{j}}^{(j)}\right)_{\mathfrak{U}} \in(E)_{\mathfrak{U}}$. If $P$ is an $n$-homogeneous continuous polynomial and $A$ is its associated symmetric $n$-linear mapping, the iterated extension, $\bar{P}$, of $P$ to $(E)_{\mathfrak{U}}$ is defined by

$$
\bar{P}\left(\left(z_{\mathfrak{i}}\right)_{\mathfrak{U}}\right):=\bar{A}\left(\left(z_{\mathfrak{i}}\right)_{\mathfrak{U}}, \ldots,\left(z_{\mathfrak{i}}\right)_{\mathfrak{U}}\right),
$$

and this coincides with $\lim _{\mathfrak{i}_{1}, \mathfrak{U}} \ldots \lim _{\mathfrak{i}_{n}, \mathfrak{U}} A\left(z_{\mathfrak{i}_{1}}, \ldots, z_{\mathfrak{i}_{n}}\right)$. 
On the other hand the uniterated extension, $\widetilde{P}$, is defined by

$$
\widetilde{P}\left(\left(z_{\mathfrak{i}}\right) \mathfrak{U}\right):=\lim _{\mathfrak{i}, \mathfrak{U}} P\left(z_{\mathfrak{i}}\right)
$$

Similarly, there are two analogous ways of extending a polynomial from a Banach space into its bidual. The classical Aron-Berner extension 1] which is, by construction, iterated (the associated multilinear form is extended from the last variable to the first one by weak-star continuity) and the uniterated AronBerner extension (a term coined in [13]). We need to construct a local ultrapower of $E$ in order to define this uniterated extension, so we give some details.

First, we recall the Principle of Local Reflexivity: for each $M \in \mathrm{FIN}\left(E^{\prime \prime}\right)$, $N \in \mathrm{FIN}\left(E^{\prime}\right)$ and $\varepsilon>0$, there exists an operator $T \in \mathcal{L}(M, E)$ such that

1. $T$ is an $\varepsilon$-isometry, that is, $(1-\varepsilon)\left\|x^{\prime \prime}\right\| \leq\left\|T\left(x^{\prime \prime}\right)\right\| \leq(1+\varepsilon)\left\|x^{\prime \prime}\right\|$.

2. $T\left(x^{\prime \prime}\right)=x^{\prime \prime}$ for every $x^{\prime \prime} \in M \cap E$.

3. $x^{\prime}\left(T\left(x^{\prime \prime}\right)\right)=x^{\prime \prime}\left(x^{\prime}\right)$ for $x^{\prime \prime} \in M$ and $x^{\prime} \in N$.

Let $I$ be the set of all triples $(M, N, \varepsilon)$, where $M$ and $N$ are finite-dimensional subspaces of $E^{\prime \prime}$ and $E^{\prime}$ respectively and $\varepsilon>0$. For each $\mathfrak{i} \in I$, we denote by $M_{\mathfrak{i}}$, $N_{\mathfrak{i}}$ and $\varepsilon_{\mathfrak{i}}$ the three elements of the triple. We define an ordering on $I$ by setting $\mathfrak{i}<\mathfrak{j}$ if $M_{\mathfrak{i}} \subset M_{\mathfrak{j}}, N_{\mathfrak{i}} \subset N_{\mathfrak{j}}$ and $\varepsilon_{\mathfrak{i}}>\varepsilon_{\mathfrak{j}}$. The sets of the form $B_{\mathfrak{i}}=\{\mathfrak{j} \in I: \mathfrak{i} \leq \mathfrak{j}\}$ form a filterbase. Let $\mathfrak{U}$ be an ultrafilter on $I$ which contains this filterbase. The filter $\mathfrak{U}$ thus constructed is called a local ultrafilter for $E$, and $(E)_{\mathfrak{U}}$ is called a local ultrapower of $E$.

Finally, let us fix, for each $\mathfrak{i} \in I$, an operator $T_{\mathfrak{i}}: M_{\mathfrak{i}} \rightarrow E$ in accordance with the Principle of Local Reflexivity. The canonical embedding of $E$ into the ultrapower $(E)_{\mathfrak{U}}$ extends to a canonical embedding $J_{E}: E^{\prime \prime} \rightarrow(E)_{\mathfrak{U}}$ defined by $J_{E}\left(x^{\prime \prime}\right)=\left(x_{\mathfrak{i}}\right)$, where $x_{\mathfrak{i}}$ is equal to $T_{\mathfrak{i}}\left(x^{\prime \prime}\right)$ if $x^{\prime \prime} \in M_{\mathfrak{i}}$ and 0 otherwise. In this way, $J_{E}\left(E^{\prime \prime}\right)$ is the range of a norm one projection on $(E)_{\mathfrak{U}}$. This projection $\operatorname{Pr}:(E)_{\mathfrak{U}} \rightarrow J_{E}\left(E^{\prime \prime}\right)$ is given by $\operatorname{Pr}\left(\left(x_{\mathfrak{i}}\right)_{\mathfrak{U}}\right)=J_{E}\left(w^{*}-\lim _{\mathfrak{i}, \mathfrak{U}} x_{\mathfrak{i}}\right)$ (the weak-star limit in $E^{\prime \prime}$ of the collection $\left.\left(x_{\mathfrak{i}}\right)\right)$.

For a polynomial $P \in \mathcal{P}^{n}(E)$ its uniterated Aron-Berner extension to $E^{\prime \prime}$ is defined by $\widetilde{P} \circ J \in \mathcal{P}^{n}\left(E^{\prime \prime}\right)$.

Let us recall some definitions from the theory of Banach polynomial ideals [16. A Banach ideal of continuous scalar valued $n$-homogeneous polynomials is a pair $\left(\mathcal{Q},\|\cdot\|_{\mathcal{Q}}\right)$ such that:

(i) $\mathcal{Q}(E)=\mathcal{Q} \cap \mathcal{P}^{n}(E)$ is a linear subspace of $\mathcal{P}^{n}(E)$ and $\|\cdot\|_{\mathcal{Q}}$ is a norm which makes the pair $\left(\mathcal{Q},\|\cdot\|_{\mathcal{Q}}\right)$ a Banach space. 
(ii) If $T \in \mathcal{L}\left(E_{1}, E\right), P \in \mathcal{Q}(E)$ then $P \circ T \in \mathcal{Q}\left(E_{1}\right)$ and

$$
\|P \circ T\|_{\mathcal{Q}\left(E_{1}\right)} \leq\|P\|_{\mathcal{Q}(E)}\|T\|^{n} .
$$

(iii) $z \mapsto z^{n}$ belongs to $\mathcal{Q}(\mathbb{K})$ and has norm 1 .

Let $\left(\mathcal{Q},\|\cdot\|_{\mathcal{Q}}\right)$ be the Banach ideal of continuous scalar valued $n$-homogeneous polynomials and, for $P \in \mathcal{P}^{n}(E)$, define $\|P\|_{\mathcal{Q}^{\max }(E)}:=\sup \left\{\left\|\left.P\right|_{M}\right\|_{\mathcal{Q}(M)}: M \in\right.$ $\operatorname{FIN}(E)\} \in[0, \infty]$. The maximal kernel of $\mathcal{Q}$ is the ideal given by $\mathcal{Q}^{\max }:=\{P \in$ $\left.\mathcal{P}^{n}:\|P\|_{\mathcal{Q}^{\max }}<\infty\right\}$. An ideal $\mathcal{Q}$ is said to be maximal if $\mathcal{Q} \stackrel{1}{=} \mathcal{Q}^{\max }$.

The minimal kernel of $\mathcal{Q}$ is defined as the composition ideal $\mathcal{Q}^{\text {min }}:=\mathcal{Q} \circ \overline{\mathfrak{F}}$, where $\overline{\mathfrak{F}}$ stands for the ideal of approximable operators. In other words, a polynomial $P$ belongs to $\mathcal{Q}^{\min }(E)$ if it admits a factorization

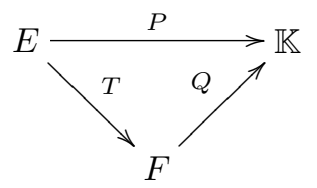

where $F$ is a Banach space, $T: E \rightarrow F$ is an approximable operator and $Q$ is in $\mathcal{Q}(F)$. The minimal norm is given by $\|P\|_{\mathcal{Q}^{\min }}:=\inf \left\{\|Q\|_{\mathcal{Q}(F)}\|T\|^{n}\right\}$, where the infimum runs over all possible factorizations as in (2.1). An ideal $\mathcal{Q}$ is said to be minimal if $\mathcal{Q} \stackrel{1}{=} \mathcal{Q}^{\text {min }}$.

For properties of maximal and minimal ideals of homogeneous polynomials and examples see [14, 17] and the references therein.

Floret and Hunfeld proved the following result [17, Theorems 3.2 and 3.3].

Theorem 2.2. Let $\mathcal{Q}$ be a maximal ideal of $n$-homogeneous polynomials, $Q \in$ $\mathcal{P}^{n}(E)$ and $\mathfrak{U}$ an ultrafilter of $E$. Then $P$ belongs to $\mathcal{Q}(E)$ if and only if the uniterated extension $\widetilde{P}$ is in $\mathcal{Q}\left((E)_{\mathfrak{U}}\right)$. In this case $\|\widetilde{P}\|_{\mathcal{Q}\left((E)_{\mathfrak{L}}\right)}=\|P\|_{\mathcal{Q}(E)}$.

If $\mathfrak{U}$ is a local ultrafilter for $E$ then we also have that $P$ belongs to $\mathcal{Q}(E)$ if and only if the uniterated Aron-Berner extension $\widetilde{P} \circ J$ belongs to $\mathcal{Q}\left(E^{\prime \prime}\right)$, and moreover $\|\widetilde{P} \circ J\|_{\mathcal{Q}\left(E^{\prime \prime}\right)}=\|P\|_{\mathcal{Q}(E)}$.

We will present a similar theorem for the iterated extension to the ultrapower and for the Aron-Berner extension. We will also conclude that the same holds in the case that $\mathcal{Q}$ is a minimal ideal of homogeneous polynomials.

The following proposition is due to Lindström and Ryan 20, Proposition 2.1]. It states that the Aron-Berner extension can be recovered from the iterated extension to a local ultrapower of $E$ : 
Proposition 2.3. If $(E)_{\mathfrak{U}}$ is a local ultrapower of $E$, then the restriction of $\bar{P}$ to the canonical image of $E^{\prime \prime}$ in $(E)_{\mathfrak{U}}$ coincides with the Aron-Berner extension of $P$ to $E^{\prime \prime}$.

\section{$\S 3$. The results}

Maximal and minimal ideals of homogeneous polynomials are easily seen to be closed under the Aron-Berner extension: just use a multilinear version of the Extension Lemma [9, 13.2] (whose proof is identical) and the main result of [15]. In this section we will show that this extension is actually an isometry.

First, let $A$ be the symmetric multilinear form associated to a polynomial $P$. For each fixed $j, 1 \leq j \leq n, x_{1}, \ldots, x_{j-1} \in E$, and $z_{j}, z_{j+1}, \ldots z_{n} \in(E)_{\mathfrak{U}}$, we have

$$
\begin{aligned}
\bar{A}\left(h_{E} x_{1}, \ldots, h_{E} x_{j-1}, z_{j}, z_{j+1}, \ldots, z_{n}\right) & \\
& =\lim _{i_{j}, \mathfrak{U}} \bar{A}\left(h_{E} x_{1}, \ldots, h_{E} x_{j-1}, h_{E} z_{\mathfrak{i}_{j}}^{(j)}, z_{j+1}, \ldots, z_{n}\right),
\end{aligned}
$$

where $\bar{A}$ is the iterated extension of $A$ to a local ultrapower.

Now, we will imitate the procedure used by Davie and Gamelin in [8]. Denote by $A$ the symmetric $n$-linear form associated to $P$. We have the following lemma:

Lemma 3.1. Let $M \in \mathrm{FIN}\left((E)_{\mathfrak{U}}\right)$ and $z_{1}, \ldots, z_{r} \in M$. For a given natural number $m$ and $\varepsilon>0$ there exist operators $R_{1}, \ldots, R_{m} \in \mathcal{L}(M, E)$ with norm less than or equal to 1 such that

$$
\left|A\left(R_{i_{1}} z_{k}, \ldots, R_{i_{n}} z_{k}\right)-\bar{A}\left(z_{k}, \ldots, z_{k}\right)\right|<\varepsilon
$$

for any distinct indices $i_{1}, \ldots, i_{n}$ between 1 and $m$ and every $k=1, \ldots, r$.

Proof. Since $A$ is symmetric, in order to prove the lemma it suffices to obtain (3.1) for $i_{1}<\cdots<i_{n}$. We will select the operators $R_{1}, \ldots, R_{m}$ inductively by the following procedure. By Proposition 2.1, for each $\mathfrak{i} \in I$ there exists an operator $R_{\mathfrak{i}} \in \mathcal{L}(M, E)$ with norm less than or equal to 1 such $z_{k}=\left(R_{\mathfrak{i}} z_{k}\right)_{\mathfrak{U}}$.

Since $z_{k}=\left(R_{\mathfrak{i}} z_{k}\right)_{\mathfrak{U}}$ for each $k$, the set $\left\{\mathfrak{i} \in I: \bar{A}\left(h_{E} R_{\mathfrak{i}} z_{k}, z_{k}, \ldots, z_{k}\right)-\right.$ $\left.\bar{A}\left(z_{k}, z_{k}, \ldots, z_{k}\right) \mid<\varepsilon / n\right\}$ belongs to the filter $\mathfrak{U}$. Therefore, we can pick $R_{1} \in$ $\mathcal{L}(M, E)$ such that

$$
\left|\bar{A}\left(h_{E} R_{1} z_{k}, z_{k}, \ldots, z_{k}\right)-\bar{A}\left(z_{k}, z_{k}, \ldots, z_{k}\right)\right|<\varepsilon / n
$$

for every $k=1, \ldots, r$.

In a similar way we can choose $R_{2}$ such that

$$
\left|\bar{A}\left(h_{E} R_{2} z_{k}, z_{k}, \ldots, z_{k}\right)-\bar{A}\left(z_{k}, z_{k}, \ldots, z_{k}\right)\right|<\varepsilon / n,
$$


and moreover,

$$
\left|\bar{A}\left(h_{E} R_{1} z_{k}, h_{E} R_{2} z_{k}, z_{k}, \ldots, z_{k}\right)-\bar{A}\left(h_{E} R_{1} z_{k}, z_{k}, \ldots, z_{k}\right)\right|<\varepsilon / n
$$

for every $k$. Proceeding in this way, we get $R_{l}$ 's so that

$$
\begin{aligned}
\mid \bar{A}\left(h_{E} R_{i_{1}} z_{k}, \ldots, h_{E} R_{i_{r-1}} z_{k}, h_{E} R_{i_{r}} z_{k}, z_{k}, \ldots, z_{k}\right) \\
-\bar{A}\left(h_{E} R_{i_{1}} z_{k}, \ldots, h_{E} R_{i_{r-1}} z_{k}, z_{k}, \ldots, z_{k}\right) \mid<\varepsilon / n
\end{aligned}
$$

whenever $i_{1}<\cdots<i_{r}$ and $k=1, \ldots, r$. Then

$$
\left|\bar{A}\left(h_{E} R_{i_{1}} z_{k}, \ldots, h_{E} R_{i_{n}} z_{k}\right)-\bar{A}\left(z_{k} \ldots, z_{k}\right)\right|
$$

is estimated by the sum of $n$ terms

$$
\begin{aligned}
\mid \bar{A}\left(h_{E} R_{i_{1}} z_{k}, \ldots, h_{E} R_{i_{n}} z_{k}\right)-\bar{A} & \left(h_{E} R_{i_{1}} z_{k}, \ldots, h_{E} R_{i_{n-1}} z_{k}, z_{k}\right) \mid+\cdots \\
& +\left|\bar{A}\left(h_{E} R_{i_{1}} z_{k}, z_{k} \ldots, z_{k}\right)-\bar{A}\left(z_{k}, \ldots, z_{k}\right)\right|
\end{aligned}
$$

each smaller than $\varepsilon / n$, for all $k=1, \ldots, r$.

Proposition 3.2. Let $M \in \operatorname{FIN}\left((E)_{\mathfrak{U}}\right)$ and $z_{1}, \ldots, z_{r} \in M, P: E \rightarrow \mathbb{K}$ a continuous polynomial and $\varepsilon>0$. There exist a finite subset $\mathfrak{F}$ of $\mathbb{N}$ and operators $\left(R_{i}\right)_{i \in \mathfrak{F}}$ in $\mathcal{L}(M, E)$ with norm less than or equal to 1 such that

$$
\left|\sum_{k=1}^{r} \bar{P}\left(z_{k}\right)-\sum_{k=1}^{r} P\left(\frac{1}{|\mathfrak{F}|} \sum_{i \in \mathfrak{F}} R_{i} z_{k}\right)\right|<\varepsilon .
$$

Proof. For $\varepsilon>0$, fix $m$ large enough and choose $R_{1}, \ldots, R_{m}$ as in the previous lemma, such that

$$
\left|\bar{A}\left(z_{k}, \ldots, z_{k}\right)-A\left(R_{i_{1}} z_{k}, \ldots, R_{i_{n}} z_{k}\right)\right|<\varepsilon / 2 r
$$

for any distinct indices $i_{1}, \ldots, i_{n}$ between 1 and $m$ and every $k=1, \ldots, r$. Now, we set $\mathfrak{F}=\{1, \ldots, m\}$ and define $R \in \mathcal{L}(M, E)$ by $R:=\frac{1}{|\mathfrak{F}|} \sum_{i \in \mathfrak{F}} R_{i}$. For $k \in$ $\{1, \ldots, r\}$, we have

$$
\begin{aligned}
\left|\bar{P}\left(z_{k}\right)-P\left(R z_{k}\right)\right| & =\left|\frac{1}{m^{n}} \sum_{i_{1}, \ldots, i_{n}=1}^{m}\left[\bar{A}\left(z_{k}, \ldots, z_{k}\right)-A\left(R_{i_{1}} z_{k}, \ldots, R_{i_{n}} z_{k}\right)\right]\right| \\
& \leq\left|\Sigma_{1}^{k}\right|+\left|\Sigma_{2}^{k}\right|
\end{aligned}
$$

where $\Sigma_{1}^{k}$ is the sum over the $n$-tuples of non-repeated indices (which is less than $\varepsilon / 2 r)$ and $\Sigma_{2}^{k}$ is the sum over the remaining indices. It is easy to show that there 
are exactly $m^{n}-\prod_{j=0}^{n-1}(m-j)$ summands in $\Sigma_{2}^{k}$, each bounded by a constant $C>0$ (obviously we can assume that $C$ is independent of $k$ ), thus

$$
\left|\Sigma_{2}^{k}\right| \leq \frac{1}{m^{n}}\left(m^{n}-\prod_{j=0}^{n-1}(m-j)\right) C=\left[1-\left(1-\frac{1}{m}\right) \ldots\left(1-\frac{n-1}{m}\right)\right] C .
$$

For $m$ sufficiently large this is less than $\varepsilon / 2 r$.

Recall that an $s$-tensor norm $\alpha$ is called finitely generated if for every Banach space $E$ and $z \in \otimes^{n, s} E$, we have $\alpha\left(z, \otimes^{n, s} E\right)=\inf \left\{\alpha\left(z, \otimes^{n, s} M\right): M \in\right.$ $\left.\operatorname{FIN}(E), z \in \otimes^{n, s} M\right\}$. Now we can state the main theorem:

Theorem 3.3. Let $\alpha$ be a finitely generated s-tensor norm and $P \in\left(\widetilde{\otimes}_{\alpha}^{n, s} E\right)^{\prime}$ a polynomial. The iterated extension $\bar{P}$ of $P$ to the ultrapower $(E)_{\mathfrak{U}}$ belongs to $\left(\widetilde{\otimes}_{\alpha}^{n, s}(E)_{\mathfrak{U}}\right)^{\prime}$ and

$$
\|P\|_{\left(\widetilde{\otimes}_{\alpha}^{n, s} E\right)^{\prime}}=\|\bar{P}\|_{\left(\widetilde{\otimes}_{\alpha}^{n, s}(E)_{\mathfrak{U}}\right)^{\prime}} .
$$

Equivalently, if $\mathcal{Q}$ is a maximal ideal of $n$-homogeneous polynomials and $P \in \mathcal{Q}(E)$, the iterated extension $\bar{P}$ of $P$ to the ultrapower $(E)_{\mathfrak{U}}$ belongs to $\mathcal{Q}\left((E)_{\mathfrak{U}}\right)$ and

$$
\|P\|_{\mathcal{Q}(E)}=\|\bar{P}\|_{\mathcal{Q}\left((E)_{\mathfrak{U}}\right)} .
$$

Proof. Thanks to the representation theorem for maximal polynomial ideals [17. Section 3.2] (see also [14, Section 4]), it is enough to show the first statement.

Let $w \in \otimes^{n, s} M$, where $M \in \operatorname{FIN}\left((E)_{\mathfrak{U}}\right)$. Since $\alpha$ is finitely generated, we only have to show that

$$
|\langle\bar{P}, w\rangle| \leq\|P\|_{\left(\widetilde{\otimes}_{\alpha}^{n, s} E\right)^{\prime}} \alpha\left(w, \otimes^{n, s} M\right) .
$$

Now, $w=\sum_{k=1}^{r} \otimes^{n} z_{k}$ with $z_{k} \in M$. Given $\varepsilon>0$, by Proposition 3.2 we can take a finite set $\mathfrak{F}$ and operators $\left(R_{i}\right)_{i \in \mathfrak{F}}$ with $\left\|R_{i}\right\|_{\mathcal{L}(M, E)} \leq 1$ such that

$$
\left|\sum_{k=1}^{r} \bar{P}\left(z_{k}\right)-\sum_{k=1}^{r} P\left(\frac{1}{|\mathfrak{F}|} \sum_{i \in \mathfrak{F}} R_{i} z_{k}\right)\right|<\varepsilon
$$

Therefore,

$$
\begin{aligned}
|\langle\bar{P}, w\rangle| & =\left|\sum_{k=1}^{r} \bar{P}\left(z_{k}\right)\right| \\
& \leq\left|\sum_{k=1}^{r} \bar{P}\left(z_{k}\right)-\sum_{k=1}^{r} P\left(\frac{1}{|\mathfrak{F}|} \sum_{i \in \mathfrak{F}} R_{i} z_{k}\right)\right|+\left|\sum_{k=1}^{r} P\left(\frac{1}{|\mathfrak{F}|} \sum_{i \in \mathfrak{F}} R_{i} z_{k}\right)\right| \\
& \leq \varepsilon+\left|\left\langle P, \sum_{k=1}^{r} \otimes^{n} \frac{1}{|\mathfrak{F}|} \sum_{i \in \mathfrak{F}} R_{i} z_{k}\right\rangle\right|
\end{aligned}
$$




$$
\begin{aligned}
& \leq \varepsilon+\|P\|_{\left(\widetilde{\otimes}_{\alpha}^{n, s} E\right)^{\prime}} \alpha\left(\sum_{k=1}^{r} \otimes^{n} \frac{1}{|\mathfrak{F}|} \sum_{i \in \mathfrak{F}} R_{i} z_{k}, \otimes^{n, s} E\right) \\
& \leq \varepsilon+\|P\|_{\left(\widetilde{\otimes}_{\alpha}^{n, s} E\right)^{\prime}} \alpha\left(\otimes^{n, s} R\left(\sum_{k=1}^{r} z_{k}\right), \otimes^{n, s} E\right),
\end{aligned}
$$

where $R=\frac{1}{|\mathfrak{F}|} \sum_{i \in \mathfrak{F}} R_{i}$ (note that $\|R\|_{\mathcal{L}(M, E)} \leq 1$ since each $\left\|R_{i}\right\|_{\mathcal{L}(M, E)} \leq 1$ ). By the metric mapping property of $\alpha$ and the previous inequality we get

$$
|\langle\bar{P}, w\rangle| \leq \varepsilon+\|P\|_{\left(\widetilde{\otimes}_{\alpha}^{n, s} E\right)^{\prime}} \alpha\left(\sum_{k=1}^{r} \otimes^{n} z_{k}, \otimes^{n, s} M\right),
$$

which ends the proof.

The following result can be seen as a symmetric version of the Extension Lemma [9, 13.2].

Corollary 3.4. Let $\alpha$ be a finitely generated s-tensor norm of order $n$ and $P \in$ $\left(\widetilde{\otimes}_{\alpha}^{n, s} E\right)^{\prime}$ be a polynomial. Then the Aron-Berner extension $\mathrm{AB}(P)$ of $P$ is in $\left(\widetilde{\otimes}_{\alpha}^{n, s} E^{\prime \prime}\right)^{\prime}$ and

$$
\|P\|_{\left(\widetilde{\otimes}_{\alpha}^{n, s} E\right)^{\prime}}=\|\mathrm{AB}(P)\|_{\left(\widetilde{\otimes}_{\alpha}^{n, s} E^{\prime \prime}\right)^{\prime}} \cdot
$$

Therefore, if $\mathcal{Q}$ is a maximal ideal of $n$-homogeneous polynomials and we take $P \in \mathcal{Q}(E)$, then its Aron-Berner extension is in $\mathcal{Q}\left(E^{\prime \prime}\right)$ and

$$
\|P\|_{\mathcal{Q}(E)}=\|\mathrm{AB}(P)\|_{\mathcal{Q}\left(E^{\prime \prime}\right)} .
$$

Proof. Let $(E)_{\mathfrak{U}}$ be a local ultrapower of $E$ and $J_{E}: E^{\prime \prime} \rightarrow(E)_{\mathfrak{U}}$ the canonical embedding. By Proposition 2.3 the iterated extension to the local ultrapower of $E$ restricted to $E^{\prime \prime}$ coincides with the Aron-Berner extension of $P$. In other words, $\mathrm{AB}(P)=\bar{P} \circ J_{E}$. Therefore,

$$
\|\mathrm{AB}(P)\|_{\mathcal{Q}\left(E^{\prime \prime}\right)}=\left\|\bar{P} \circ J_{E}\right\|_{\mathcal{Q}\left(E^{\prime \prime}\right)} \leq\|\bar{P}\|_{\mathcal{Q}\left((E)_{\mathfrak{U}}\right)}\left\|J_{E}\right\|^{n}=\|P\|_{\mathcal{Q}(E)} .
$$

The other inequality is immediate.

As a direct consequence we also obtain a symmetric version of the Embedding Lemma [9, 13.3]:

Corollary 3.5. The natural maps

$$
\begin{aligned}
& \otimes^{n, s} J_{E}: \otimes_{\alpha}^{n, s} E \rightarrow \otimes_{\alpha}^{n, s}(E)_{\mathfrak{U}}, \\
& \otimes^{n, s} \kappa_{E}: \otimes_{\alpha}^{n, s} E \rightarrow \otimes_{\alpha}^{n, s} E^{\prime \prime}
\end{aligned}
$$

are isometries for every finitely generated tensor norm $\alpha$. 
Proof. Clearly $\left\|\otimes^{n, s} J_{E}\right\| \leq 1$, and $\langle P, w\rangle=\left\langle\bar{P}, \otimes^{n, s} J_{E} w\right\rangle$ gives the remaining inequality. Similarly we infer that $\otimes^{n, s} \kappa_{E}$ is an isometry.

Now we turn our attention to minimal ideals of polynomials. In order to show that the Aron-Berner extension is also an isometry for minimal ideals, we first need the following simple result:

Lemma 3.6. Let $T: E \rightarrow F$ be an approximable operator. Then $(T)_{\mathfrak{U}}:(E)_{\mathfrak{U}} \rightarrow$ $(F)_{\mathfrak{U}}$ is also approximable.

Proof. It is sufficient to show that if $T: E \rightarrow F$ is a rank-one operator, then so too is $(T)_{\mathfrak{U}}$. Let $x^{\prime} \in E^{\prime}$ be such that $T(x)=x^{\prime}(x) y$. If we denote $\lambda:=\lim _{i, \mathfrak{U}} x^{\prime}\left(x_{i}\right)$ we see easily that $(T)_{\mathfrak{U}}\left(x_{i}\right)_{\mathfrak{U}}=\left(x^{\prime}\left(x_{i}\right) y\right)_{\mathfrak{U}}=\lambda h_{F} y$.

Theorem 3.7. Let $\mathcal{Q}$ be a minimal ideal. If $P \in \mathcal{Q}(E)$, then the iterated extension $\bar{P}$ belongs to $\mathcal{Q}\left((E)_{\mathfrak{U}}\right)$ and

$$
\|P\|_{\mathcal{Q}(E)}=\|\bar{P}\|_{\mathcal{Q}\left((E)_{\mathfrak{U}}\right)}
$$

Proof. Since $P \in \mathcal{Q}(E) \stackrel{1}{=}\left(\left(\mathcal{Q}^{\max }\right)^{\min }\right)(E)$ (see [14, 3.4]), given $\varepsilon>0$ there exist a Banach space $F$, an approximable operator $T: E \rightarrow F$ and a polynomial $Q \in$ $\mathcal{Q}^{\max }(F)$ such that $P=Q \circ T$ and $\|Q\|_{\mathcal{Q}^{\max (F)}}\|T\|^{n} \leq\|P\|_{\mathcal{Q}(E)}+\varepsilon$ (as in (2.1)). Notice that $\bar{P}=\bar{Q} \circ(T)_{\mathfrak{U}}$. By Theorem 3.3 we have $\|Q\|_{\mathcal{Q}^{\max (F)}}=\|\bar{Q}\|_{\mathcal{Q}^{\max }((F) \mathfrak{U})}$. Since by Lemma 3.6. $(T)_{\mathfrak{U}}$ is also approximable, we conclude that

$$
\|\bar{P}\|_{\mathcal{Q}\left((E)_{\mathfrak{U}}\right)} \leq\|\bar{Q}\|_{\mathcal{Q}^{\max }\left((F)_{\mathfrak{U}}\right)}\left\|(T)_{\mathfrak{U}}\right\|^{n}=\|Q\|_{\mathcal{Q}^{\max }(F)}\|T\|^{n} \leq\|P\|_{\mathcal{Q}(E)}+\varepsilon .
$$

The reverse inequality is immediate.

Similarly to Corollary 3.4 we have:

Corollary 3.8. Let $\mathcal{Q}$ be a minimal ideal. For $P \in \mathcal{Q}(E)$, its Aron-Berner extension $\mathrm{AB}(P)$ belongs to $\mathcal{Q}\left(E^{\prime \prime}\right)$ and

$$
\|P\|_{\mathcal{Q}(E)}=\|\mathrm{AB}(P)\|_{\mathcal{Q}\left(E^{\prime \prime}\right)} .
$$

We end this note with some comments on the extension of analytic functions associated to polynomial ideals.

The concept of holomorphy type was introduced by Nachbin in 23. (see also [11]). The most natural holomorphy types can be seen as sequences of polynomial ideals $\mathcal{Q}=\left\{\mathcal{Q}_{k}\right\}_{k}\left(\mathcal{Q}_{k}\right.$ is an ideal of polynomials of degree $\left.k, k=1,2, \ldots\right)$, where some kind of affinity between ideals of different degrees is necessary [3, 6]. In [5], given such a sequence of polynomial ideals, an associated Fréchet space of entire functions is defined. In [22, the corresponding definition for analytic functions defined on the unit ball of a Banach is given: 
Definition 3.9. Let $\mathcal{Q}=\left\{\mathcal{Q}_{k}\right\}_{k}$ be a sequence of polynomial ideals and $E$ be a Banach space. The space of $\mathcal{Q}$-holomorphic functions of bounded type on $B_{E}$ is defined as

$$
H_{b \mathcal{Q}}\left(B_{E}\right)=\left\{f \in H\left(B_{E}\right): \frac{d^{k} f(0)}{k !} \in \mathcal{Q}_{k}(E) \text { and } \lim _{k \rightarrow \infty}\left\|\frac{d^{k} f(0)}{k !}\right\|_{\mathcal{Q}_{k}(E)}^{1 / k}<1\right\} .
$$

An example of this kind of space is that of boundedly-integral holomorphic functions in the ball $H_{b I}$, studied in [10].

An immediate consequence of our results is the following: let $\mathcal{Q}=\left\{\mathcal{Q}_{k}\right\}_{k}$ be a sequence of polynomial ideals, each $\mathcal{Q}_{k}$ being either maximal or minimal. If $E$ is a Banach space, then a holomorphic function $f$ belongs to $H_{b \mathcal{Q}}\left(B_{E}\right)$ if and only if its Aron-Berner extension belongs to $H_{b \mathcal{Q}}\left(B_{E^{\prime \prime}}\right)$. Note that no coherence between ideals of different degrees is needed for this to hold.

\section{Acknowledgements}

The first author was partially supported by ANPCyT PICT 05 17-33042, UBACyT Grant X038 and ANPCyT PICT 06 00587. The second author was partially supported by ANPCyT PICT 05 17-33042, UBACyT Grant X863 and a Doctoral fellowship from CONICET.

\section{References}

[1] R. M. Aron and P. D. Berner, A Hahn-Banach extension theorem for analytic mappings, Bull. Soc. Math. France 106 (1978), 3-24. Zbl 0378.46043 MR 0508947

[2] R. M. Aron, P. Galindo, D. García, and M. Maestre, Regularity and algebras of analytic functions in infinite dimensions, Trans. Amer. Math. Soc. 348 (1996), 543-559. Zbl 0844.46024 MR 1340167

[3] G. Botelho, H.-A. Braunss, H. Junek, and D. Pellegrino, Holomorphy types and ideals of multilinear mappings, Studia Math. 177 (2006), 43-65. Zbl 1112.46038 MR 2283707

[4] D. Carando, Extendible polynomials on Banach spaces, J. Math. Anal. Appl. 233 (1999), 359-372. Zbl 0942.46029 MR 1684392

[5] D. Carando, V. Dimant, and S. Muro, Hypercyclic convolution operators on Fréchet spaces of analytic functions, J. Math. Anal. Appl. 336 (2007), 1324-1340. Zbl 1128.47005 MR 2353017

[6] Coherent sequences of polynomial ideals on Banach spaces, Math. Nachr. 282 (2009), 1111-1133. Zbl 1181.47076 MR 2547712

[7] D. Carando and I. Zalduendo, A Hahn-Banach theorem for integral polynomials, Proc. Amer. Math. Soc. 127 (1999), 241-250. Zbl 0908.46031 MR 1458865

[8] A. M. Davie and T. W. Gamelin, A theorem on polynomial-star approximation, Proc. Amer. Math. Soc. 106 (1989), 351-356. Zbl 0683.46037 MR 0947313

[9] A. Defant and K. Floret, Tensor norms and operator ideals, North-Holland Math. Stud.. 176, North-Holland, Amsterdam, 1993. Zbl 0774.46018 MR 1209438

[10] V. Dimant, P. Galindo, M. Maestre, and I. Zalduendo, Integral holomorphic functions, Studia Math. 160 (2004), 83-99. Zbl 1055.46025 MR 2029740 
[11] S. Dineen, Holomorphy types on a Banach space, Studia Math. 39 (1971), 241-288. Zbl 0235.32013 MR 0304705

[12] S. Dineen and R. M. Timoney, Complex geodesics on convex domains, in Progress in functional analysis, Proc. Int. Meet. Occas. 60th Birthd. M. Valdivia (Peñíscola, 1990), NorthHolland Math. Stud. 170, North-Holland, 1992, 333-365. Zbl 0785.46044 MR 1150757

[13] K. Floret, Natural norms on symmetric tensor products of normed spaces, Note Mat. 17 (1997), 153-188. Zbl 0961.46013 MR 1749787

[14] Minimal ideals of $n$-homogeneous polynomials on Banach spaces, Results Math. 39 (2001), 201-217. Zbl 1017.46030 MR 1834571

[15] - The extension theorem for norms on symmetric tensor products of normed spaces, in Recent progress in functional analysis, North-Holland Math. Stud. 189, Elsevier, 2001, 225-237. Zbl 1010.46019 MR 1861759

[16] _ On ideals of $n$-homogeneous polynomials on Banach spaces, in Topological algebras with applications to differential geometry and mathematical physics, P. Strantzalos et al. (eds.), Univ. of Athens, 2002, 19-38. Zbl 1095.46026 MR 2000732

[17] K. Floret and S. Hunfeld, Ultrastability of ideals of homogeneous polynomials and multilinear mappings on Banach spaces, Proc. Amer. Math. Soc. 130 (2002), 1425-1435. Zbl 1027.46054 MR 1879966

[18] S. Heinrich, Ultraproducts in Banach space theory, J. Reine Angew. Math. 313 (1980), 72-104. Zbl 0412.46017 MR 0552464

[19] K. D. Kürsten, s-Zahlen und Ultraprodukte von Operatoren in Banachräumen, Doctoral Thesis, Leipzig, 1976.

[20] M. Lindström and R. A. Ryan, Applications of ultraproducts to infinite dimensional holomorphy, Math. Scand. 71 (1992), 229-242. Zbl 0845.46022 MR 1212706

[21] L. A. Moraes, The Hahn-Banach extension theorem for some spaces of n-homogeneous polynomials, in Functional analysis: surveys and recent results III (Paderborn, 1983), NorthHolland Math. Stud. 90, North-Holland, 1984, 265-274. Zbl 0553.46005 MR 0761386

[22] S. Muro, Funciones holomorfas de tipo acotado e ideales de polinomios homogéneos en espacios de Banach, Doctoral Thesis, Universidad de Buenos Aires, 2010.

[23] L. Nachbin, Topology on spaces of holomorphic mappings, Springer, Berlin, 1969. Zbl 0172.39902 MR 0254579

[24] I. Zalduendo, A canonical extension for analytic functions on Banach spaces, Trans. Amer. Math. Soc. 320 (1990), 747-763. Zbl 0709.46027 MR 1001952 